\title{
Internet of Things based Smart Electricity Meters
}

\author{
Shikha Rastogi \\ Bharati Vidyapeeth's College \\ of Engineering, New Delhi
}

\author{
Manisha Sharma \\ Bharati Vidyapeeth's College \\ of Engineering, New Delhi
}

\author{
Pratibha Varshney \\ Bharati Vidyapeeth's College \\ of Engineering, New Delhi
}

\begin{abstract}
Since energy sources are limited and it has become our need to save as much energy as possible. In this scenario, Smart meter comes into the picture. A Smart meter captures unit consumed in a specific time frame, display results and consequently provide real time inputs to the billing unit. The use of such smart meters has been growing rapidly in recent years. In fact, certain market observers estimate the global market for smart meters will accelerate from $\$ 4$ billion in 2011 to approximately $\$ 20$ billion in 2018.[1] Direct U.S. exports of smart meters also have shown solid growth in recent years, although from a small base, rising from an estimated \$180 million to \$240 million during 2008-13[2]. Smart meters are far better than those electromechanical meters used previously by the customers.
\end{abstract}

In this paper, we are explaining the concept of smart meters, different types of communication, communication employing PLC, security and frauds detection, cost optimization and data sets.

\section{General Terms}

Internet of Things, Smart metering, Network model.

\section{Keywords}

IoT, P2P, IRM, Electromechanical, PLC, AMI, MDM.

\section{INTRODUCTION}

With the great developments in the field of Internet and technologies, everything has become digital. Internet has become an important part of our lives. A new technology has entered into this picture known as Internet of Things (IoT). Internet of Things is a network comprises of many electronic devices and sensors which are connected together to exchange some information over the web. The devices based on IoT seem talking and sharing data with each other.

Smart Meter is one of the applications of IoT. It records the consumption and sends the readings to the utility office on regular basis for monitoring and billing. For a long time, traditional electromechanical meters have been used (see Figure 1).

Meter readings were noted down on the monthly basis. But now with the evolution of smart electricity meters, things are changing.

Benefits of smart meter over traditional Electromechanical meters:

1. Smart meters are less error prone. Accurate readings are obtained by the customers and utility providers.

2. Readings can be sent remotely over the web to the utility providers. Employees need not to be physically present at the site.

3. Tampering of these meters can be easily detected by the authorities.
4. Smart meters when programmed with home appliances can be used to control the electricity consumption.
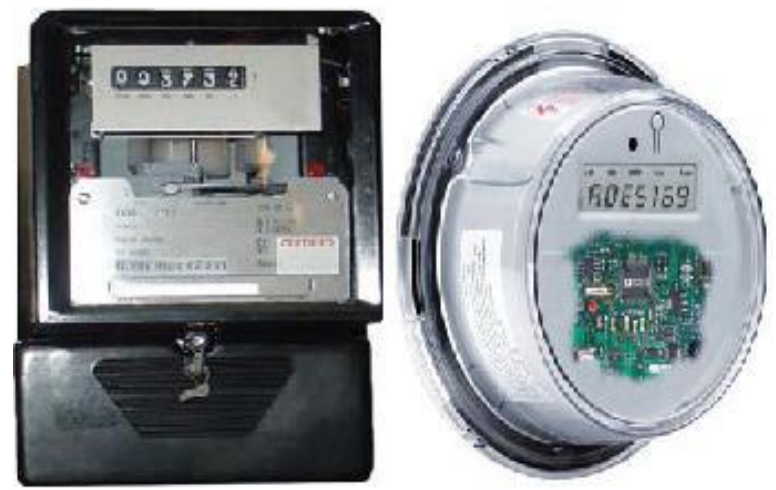

Figure 1 : Electromechanical v/s smart meter

For many years, utility providers have been concerned about the quality and economy of the energy system. However security and privacy are new emerging challenges for them [3].

\section{ARCHITECTURE OF SMART METERING SYSTEM}

There are several advantages of smart metering system like environmental and economic.

The Smart meter should have the following functionalities [4]:

1. Quantitative measurement: meter should have the capability to measure the quantity of the medium using various methods and topologies.

2. Control and Calibration: Meter should be able to compensate the small variations in the system.

3. Communication: Sending and receiving of data effectively and ability to receive upgrades from firmware.

4. Power Management: In the case when power source is not properly available meter should be able to perform its task.

5. Display: Customer should be able to see the meter readings so that he/she can control electricity consumption as well as it will be helpful in billing or payment.

6. Synchronization: Timing synchronization is very important between the meter and the utility provider's system.

So it will be beneficial to understand the architecture of this system [5] [6] (see Figure 2).

AMI: data gathered from all the meters is directed to a main server. This is called Advanced Metering Infrastructure. In 
existing AMIs with data collection intervals of few seconds to few minutes, the data collected is huge and is present in terabytes referred to as "Big Data"[7].

MDM: It is required to unify and process the data of every company at separate places. Such a centralised system is called Meter Data Management.

Data collected from meter is also useful for other subsystems like OMS (outage management System), CMS (Customer Management System), DMS (Distributed Management System) and WMS (Work Management System). Integration between these MDM and systems is done using ESB (Enterprise Service Bus).

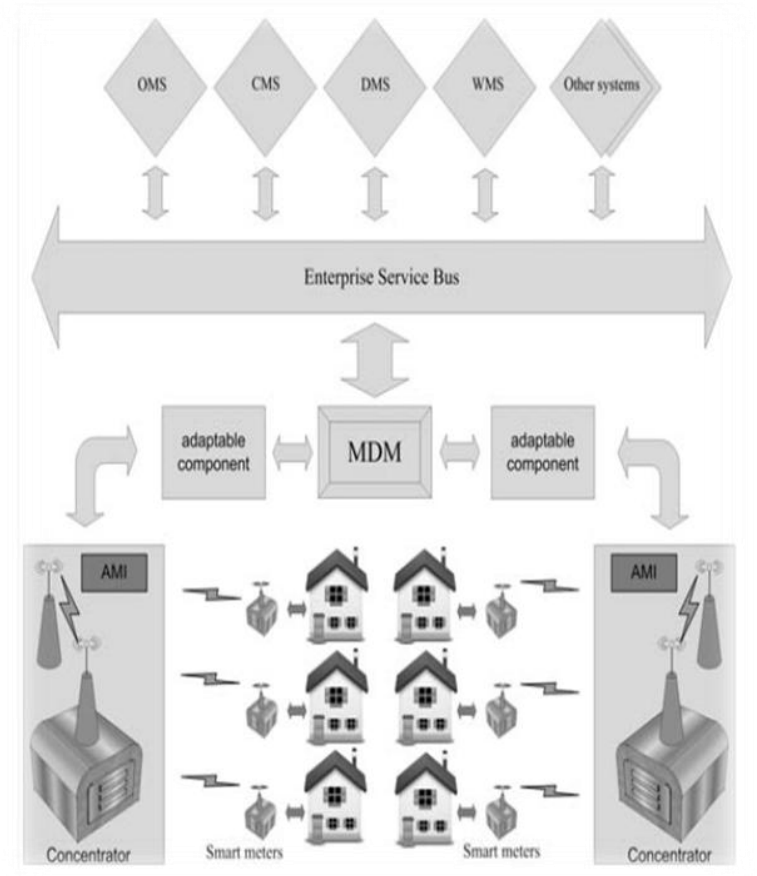

Figure 2: Architecture of smart metering system

\section{COMMUNICATION}

Smart meter should be able to communicate to the Utility providers. It should send the data read from the meter to the server of the analyser and receive the operational response. [2] There is need of a communication system that should be highly reliable and secure to transfer high volume of data. The system should be able to collect the data from all the local smart meters and send it to the authorities concerned for it [8]. Various communication technologies can be used in this system like:

- $\quad$ Power Line Carrier (PLC).

- Broadband over Power Lines (BPL).

- Copper or optical fibre.

- Cellular.

- WiMax.

- Bluetooth.

- General Packet Radio Service (GPRS).

- Internet.

- Satellite.

- P2P

- Zigbee.

Power line connection (PLC) is best to establish communication between meter and the household because it does not require a separate communication line and can be installed using power line infrastructure. In this paper, [9] the security of PLC is explained. This protocol uses public key cryptographic approach. This protocol work in two steps:

1. Check the security of the certification of the public key.

2. Hop-to-hop authentication in order to reduce the risk of Denial-Of-Service (DoS).

Components of this Protocol:

Server: The Destination to send the collected information for analysis.

Manufacturer: Manufactures the PLC Modems and IRM.

PLC Modem: It collects the meter reading from the household meters and sends them to the IRM.

IRM: It acts as an interface between PLCs and Server (see Figure 3).

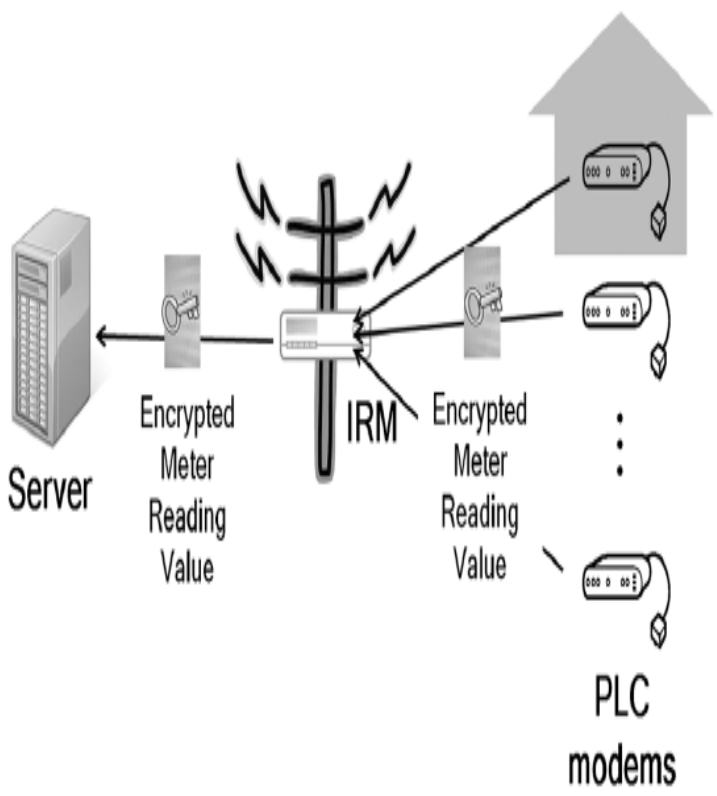

Figure 3: Network Topology of smart meter

The European Union (EU) is promoting the standardization of smart grid security as a part of smart-grid standardization throughout the project "Smart Grids: European Technology Platform," which comprised 19 detailed studies carried out at five research sections [10].

\section{Types of Attack}

COA (Ciphertext only attack): The attacker tries to deduce decryption key from the ciphertext.

KPA (Known Plaintext Attack): The attacker obtains pairs of plaintext and ciphertext by reading the meter.

CPA (Chosen plaintext Attack): The attacker can choose the plaintext and the corresponding ciphertext.

CCA (Chosen Ciphertext Attack): Since a message authentication code is generated for each encrypted meter

reading, the attacker cannot forge a valid ciphertext for an arbitrary value; however, he/she can launch this attack for public-key cryptosystems. 
Table 1 : Description of Socio-Demographic variables

\begin{tabular}{|c|c|c|c|}
\hline $\begin{array}{c}\text { Socio- } \\
\text { demographic } \\
\text { variables } \\
\end{array}$ & Description & $\begin{array}{l}\text { Number of } \\
\text { categories }\end{array}$ & Example(s) \\
\hline GSP group & $\begin{array}{c}\text { Grid supply point group in UK which are regional } \\
\text { electricity distribution networks }\end{array}$ & $\begin{array}{c}\text { Total } 14 \\
3 \text { in datasets }\end{array}$ & Southern; South Wales; North Scotland \\
\hline Age & Age of head of household & 6 & Age $26-35$ \\
\hline $\begin{array}{l}\text { Decision } \\
\text { maker type }\end{array}$ & Type of person deciding household matters & 13 & Young Couple \\
\hline $\begin{array}{c}\text { Family } \\
\text { lifestage }\end{array}$ & $\begin{array}{l}\text { The combined stage of life and family status } \\
\text { including children }\end{array}$ & 14 & Young family with children \\
\hline $\begin{array}{l}\text { Household } \\
\text { composition }\end{array}$ & $\begin{array}{l}\text { People living together and their relationships to one } \\
\text { another }\end{array}$ & 13 & Male home shares \\
\hline $\begin{array}{c}\text { Household } \\
\text { income band }\end{array}$ & Total household income per year & 10 & $£ 30,000$ to $£ 39,999$ \\
\hline $\begin{array}{l}\text { Mains gas } \\
\text { flag }\end{array}$ & $\begin{array}{l}\text { Whether a household is connected to the Main gas } \\
\text { network; if Yes, it's assumed that household uses gas }\end{array}$ & 2 & Connected to gas; not connected to gas \\
\hline $\begin{array}{l}\text { Mosaic } \\
\text { public sector } \\
\text { group } \\
\end{array}$ & $\begin{array}{c}\text { Classification on citizen's location, demographics, } \\
\text { lifestyles and behaviors }\end{array}$ & 15 & $\begin{array}{c}\text { Young, well-educated city dwellers; Wealthy } \\
\text { people living in the most sought after } \\
\text { neighborhoods }\end{array}$ \\
\hline $\begin{array}{l}\text { Mosaic } \\
\text { public sector } \\
\text { type }\end{array}$ & Subcategories of Mosaic Public Sector Group & 69 & $\begin{array}{c}\text { Young professional families settling in better } \\
\text { quality older terraces }\end{array}$ \\
\hline $\begin{array}{l}\text { Number of } \\
\text { bedrooms }\end{array}$ & Number of bedrooms of the property & 5 & $5+$ bedrooms \\
\hline Property age & When the property was built & 6 & 1871-1919 \\
\hline $\begin{array}{l}\text { Property type } \\
2011 \\
\end{array}$ & Type of property in 2011 & 5 & Purpose built flats; Farm \\
\hline $\begin{array}{l}\text { Property } \\
\text { value fine }\end{array}$ & Estimated property value & 25 & $£ 500,001$ to $£ 600,000$ \\
\hline Tenure 2011 & Property ownership in 2011 & 3 & Privately rented \\
\hline
\end{tabular}

\section{COST TYPES AND NETWORK MODEL}

Two types of costs are explained: power cost and communication cost. When large amount of meter data is sent to the server, then power cost will be reduced but since large number of channels or channel capacity will be required to send large amount of data which consequently increase the communication cost.

On the other hand, if fewer channels are leased for data sending to reduce the communication cost then some data may be lost due to congestion; hence power cost will be increased.

Several Network models have been proposed for meter data collection [11] in smart system (see Figure 4):

1. Home Area Network (HAN): The most basic network which is used to connect home appliances to the smart meter to support demand response, control energy consumption. Wi-Fi, Bluetooth etc. such technologies can be used to connect such devices in HAN. Short distances among nodes that enable low power transmission, wireless technologies are solutions to HANs. These technologies include $2.4 \mathrm{GHz} \mathrm{WiFi}$, 802.11, wireless networking protocol Zigbee and HomePlug [12].

2. Neighbourhood Area Network (NAN): multiple homes act as a community and served by a Data Aggregator unit (DAU) which collect meter data from HAN via NAN.

3. Wide Area Network (WAN): It sends all the meter data collected at DAUs to the Power Management System
(PMS). 3G, Satellites etc. technologies can be used for WAN.

The smart electricity meter consumption data used in this paper were collected by the Energy Demand Research Project (EDRP) [13] [14]. This dataset contains the electricity consumption in kWh of every one of nearly 5000 domestic consumer premises (households) in the U.K., with 30-min resolution, from April 2009 to October 2010. These consumption data were recorded by the installed smart meters at 48 time points per day: 0:00, 0:30 ...23:30.

The U.K. electricity suppliers and distributors trade $30 \mathrm{~min}$ volumes of electricity (consumption), not rates of flow (power). Hence the emphasis is on consumption rather than power.

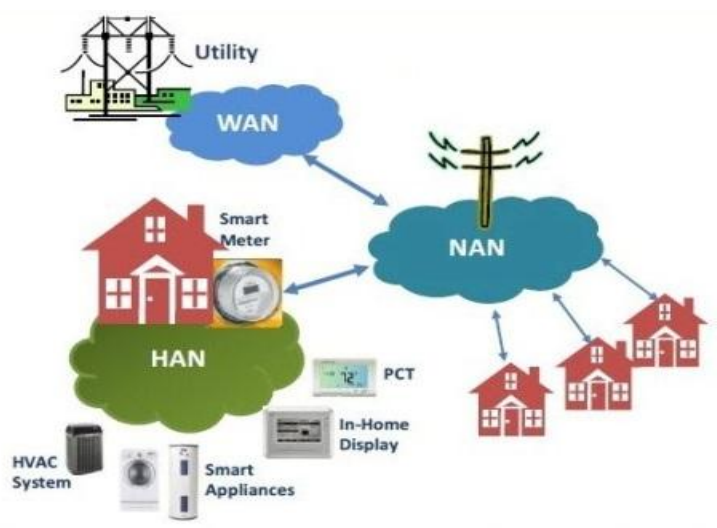

Figure 4: Network Model 
Some steps taken for the research: [15]

1. Smart meter data is obtained by local energy provider.

2. Supervisor passes this received data to the analysing team.

3. The received data contains:

a. Values from day 1 to $30 / 31$ of the month. The hourly price varies each day.

b. Smart meter consumption data values of some households.

4. After reading the data, the data is interpreted and cost, cumulative cost, overall data consumption etc. fields are calculated.

\section{CONCLUSION}

The $21^{\text {st }}$ century has brought great discoveries and advancements in the field of technology. These advancements also brought many challenges and require approaches to handle these challenges. Smart metering system is one such approach. In this paper, we explained the basic architecture of the smart metering system and a case study.

\section{ACKNOWLEDGMENTS}

Our sincere thanks to Mrs. Shikha Rastogi, our teacher for providing us her valuable guidance in developing this paper.

\section{REFERENCES}

[1] Lisa Alejandro, Caitlin Blair, Laura Blood good , Mahnaz Khan ,Martha Lawless, Daniel Meehan, Patrick Schneider ,Karl Tsuji “GLOBAL MARKET FOR SMART ELECTRICITY METERS: GOVERNMENT POLICIES DRIVING STRONG GROWTH” June 2014.

[2] Bagley, Chris. "Elster Work Done in Raleigh Moving to Mexico.” Triangle Business Journal. January 31, 2013.

[3] Ramyar Rashed Mohassel, Alan Fung, Farah Mohammadi, Kaamran Raahemifar "ELECTRICAL POWER AND ENERGY SYSTEMS" Department of Electrical and Computer Engineering, Ryerson University, Toronto, ON M5B 2K3, Canada'.Sannella, M. J. 1994 Constraint Satisfaction and Debugging for Interactive User Interfaces. Doctoral Thesis. UMI Order Number: UMI Order No. GAX95-09398., University of Washington.

[4] Silicon Laboratories, Inc. Smart metering brings intelligence and connectivity to utilities, green energy and natural resource management. Rev.1.0. [accessed August, 2013].

[5] Lilijana Djukic Petromanjanc, Oliver Momcilovic, Livan Scepanovic "SUGGESTED ARCHITECTURE OF SMART METERING SYSTEM".

[6] VUKMIROVIC S., LUKOVIC S., ERDELJAN A., KULIC F., A solution for CIM based integration of Meter Data Management in Control Center of a power system, 2010 IEEE Workshop on Environment, Energz and Structural Monitoring Systems, Toronto, Italy, 9 September 2010.

[7] Deign J, Salazar CM. Data management and analytics for utilities. FC Business Intelligence Ltd.; 2013.

[8] National Energy Technology Laboratory for the U.S. Department of Energy. Advanced metering infrastructure, NETL modern grid strategy; 2008.

[9] Sungwook Kim, Eun Young Kwon, Myungsun Kim, Jung Hee Cheon, Seong-ho Ju, Yong-hoon Lim, and Moon-seok Choi "A SECURE SMART METERINGPROTOCOL OVER POWER LINE COMMUNICATION". IEEE Transactions On Power Delivery, Vol. 26, No. 4, October 2011.

[10] SmartGrids: European Technology Platform. [Online]. Available: http://www.smartgrids.eu

[11] PENG LI (Member, IEEE), SONG GUO (Senior Member, IEEE), AND ZIXUE CHENG (Member, IEEE) "Joint Optimization of Electricity and Communication Cost for Meter Data Collection in Smart Grid"Digital Object Identier 10.1109/TETC.2013.2273890.

[12] US Department of Energy Communications requirements of smart grid technologies; October 5, 2010.

[13] Nanlin Jin, Member, IEEE, Peter Flach, Tom Wilcox, Royston Sellman, Joshua Thumim, and Arno Knobbe "Subgroup Discovery in Smart Electricity Meter Data" IEEE TRANSACTIONS ON INDUSTRIAL INFORMATICS, VOL. 10, NO. 2, MAY 2014.

[14] G. Raw and D. Ross, "Energy demand research project," Office of Gas Elect. Markets, Tech. Rep. 60163857, 2011.

[15] Praveen Vadda, Sreerama Murthy Seelam "Smart Metering for Smart Electricity Consumption" School of Computing, Blekinge Institute of Technology, 37179 Karlskrona, Sweden May 2013. 